\title{
Development of Super-Kamiokande detector simulation based on Geant4
}

\author{
M.Harada*1, Y.Koshio ${ }^{1}$, K.Hagiwara ${ }^{1}$ \\ ${ }^{1}$ Okayama University, Okayama-shi, Okayama, 700-8530, Japan \\ E-mail: pc3a4sejds.okayama-u.ac.jp
}

The Super-Kamiokande Gadolinium (SK-Gd) project is the upgrade of the Super-Kamiokande (Super-K) detector via the introducing gadolinium sulfate. For precise measurement in SK-Gd, detailed detector simulation is needed. Currently Super-K uses Geant 3 based simulation, however, the development and the update of Geant 3 was finished.. For the next 10 years Spuer-K operation, up-to-date detector simulation is needed. Especially, for diffuse supernova neutrino background search in SK-Gd, precise neutron simulation is crucial. Therefore, we are developing Geant4 based Super-K simulation software, named 'SKG4' . We started from the construction of SuperK geometry, and a comparison with the current Super-K detector simulation has been conducted. In this proceedings, current status of the development for SKG4 and the result of the several physics simulation will be reported.

36th International Cosmic Ray Conference -ICRC2019-

July 24th - August 1st, 2019

Madison, WI, U.S.A.

${ }^{*}$ Speaker. 


\section{Introduction}

Neutrinos emitted from past core-collapse supernova (CCSN) are expected to drift in our universe. This is often called Diffuse Supernova Neutrino Background(DSNB). Detecting DSNB give a new insight about the history of universe, for example, star formation and theory of supernova. One of the aim in Super-Kamiokande (Super-K) experiment is to observe DSNB..

All types of neutrinos are emitted from CCSN and drive in the universe as DSNB. Among them, electron anti-neutrino has the largest cross section via inverse beta decay (IBD) reaction $\left(\bar{v}_{e}+p \rightarrow e^{+}+n\right)$ in the energy range around few tens MeV. Super-K focuses this event and energy region for the DSNB search.

\section{Previous DSNB search}

The DSNB search was carried out in various experiments. Of course, Super-K has been done several times. Although DSNB signal couldn't detect in all search, a lowest upper limit of DSNB flux for $E_{\bar{v}_{e}}>17.3 \mathrm{MeV}$ was derived[四]. The difficulty of search for DSNB is due to the low flux of DSNB and some background. The most serious background of traditional DSNB search is decay electron from invisible muon, which is produced by atmospheric neutrinos. This electron signal is not possible to be distinguished with positron signal from DSNB in Water-Cherenkov detector. As another problem, there is energy threshold of DSNB search. DSNB has a larger flux in the low energy region. However the energy region for DSNB search in Super-K at that time is above 18 $\mathrm{MeV}$ due to reduction of background event.

To separate the positron signal of DSNB from background channel, further search with lower energy threshold, which used delayed neutron signal $\left(E_{\bar{v}_{e}}>13.3 \mathrm{MeV}\right)$ was carried out[[]]. As a neutron signal, $2.2 \mathrm{MeV}$ gamma ray from thermal neutron capture reaction on proton were used. However, the neutron signal is very low energy for Super-K, so that detection efficiency of delayed neutron signal was about $20 \%$ and the limit of DSNB flux which is derived in this search was worse than previous search[四].

The further reduction of background and the expansion of energy region in search are needed to observation of DSNB. Therefore, SuperKamiokande-Gadolinium project(SK-Gd) is in preparation[B]]. Gd has the largest capture cross-section for thermal neutron among all stable elements. Also, the gamma rays are emitted from the reaction are totally $\sim 8 \mathrm{MeV}$, which can be easily detected by Super-K. SK-Gd will enable to detect the neutron signal with high detection efficiency, by dissolving $0.2 \% \mathrm{Gd}_{2}\left(\mathrm{SO}_{4}\right)_{3}$ in mass concentration in Super-K. Figure $\mathrm{W}$ shows the detection method of IBD by using prompt positron signal and delayed neutron signal.

Toward SK-Gd, Super-K tank refurbishment work was completed in 2018, and pure water operation has already started since the end of January 2019. Introducing Gd for Super-K will start around the end of 2020.

\section{Detector simulation}

Currently, the detector simulation software of Super-K(SKDETSIM) is based on Geant3[团]. There are some problems to use SKDETSIM in future. The most serious problem is poor extensibility of Geant3. The update of physics model in Geant3 was finished, therefore, some physics 


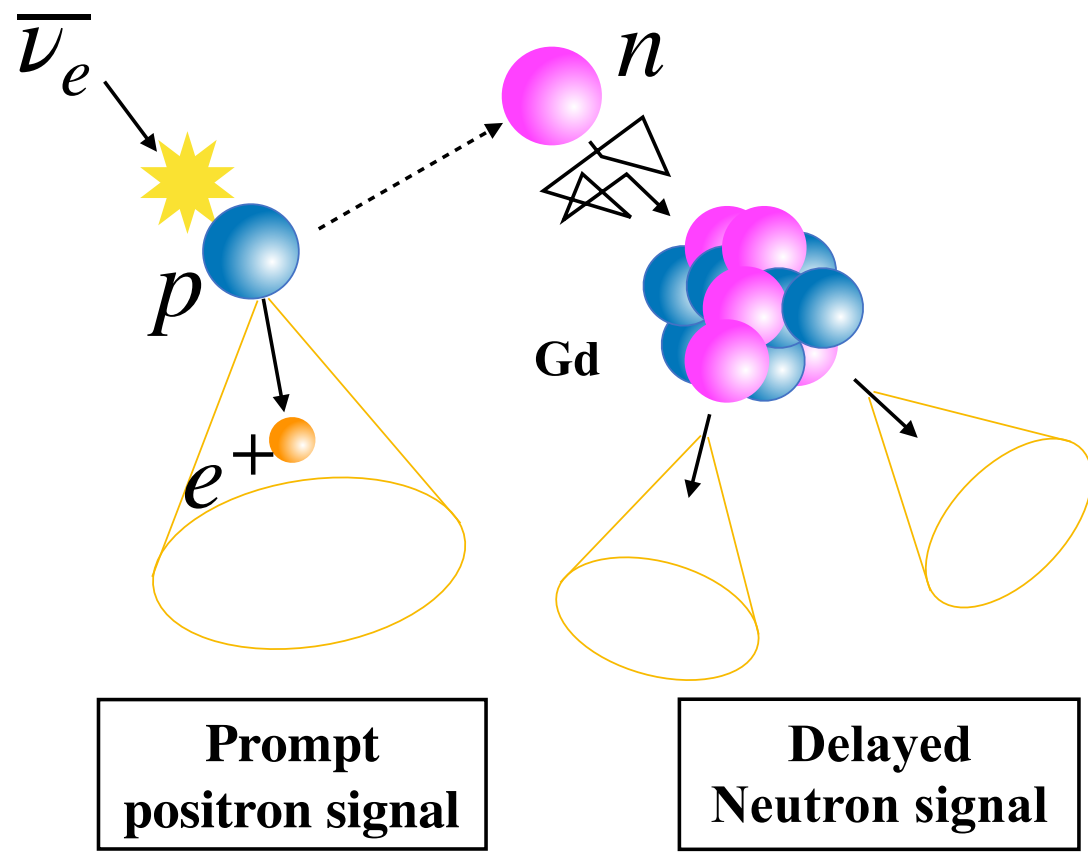

Figure 1: Schematic of inverse beta decay in SK-Gd. It will become to be possible to "Delayed coincidence" measurement.

model in SKDETSIM is not latest and these may be inaccurate. Especially, neutron interaction model is crucial for SK-Gd.

To solve these problems, new detector simulation software based on Geant4[可] was constructed, this is named "SKG4". Geant4 can use the latest physics model which is suitable for energy region of Super-K experiment, and the model based on experiment can be used. Especially, in SKG4, the model of gamma ray which is emitted from neutron capture on Gd is based on very accurate experimental result[回]. Due to these advantages, SKG4 will be possible to perform highly accurate simulation on SK-Gd.

In this proceedings, some results of comparison with SKDETSIM are reported.

\section{Detector geometry}

Detector geometry is reproduced with reference to SKDETSIM. Figure $\square$ shows the reproduced geometry. Totally 11129 photomultiplier tubes (PMTs) were installed on the wall, top, and bottom of cylindrical volume. Photoelectric surface of Super-K PMT is covered in the acrylic case.

\section{Physics process check}

The reproduction of Super-K detector geometry was already completed. Before to compare with the experimental data, the comparison of physics model between SKDETSIM and SKG4 was carried out. 


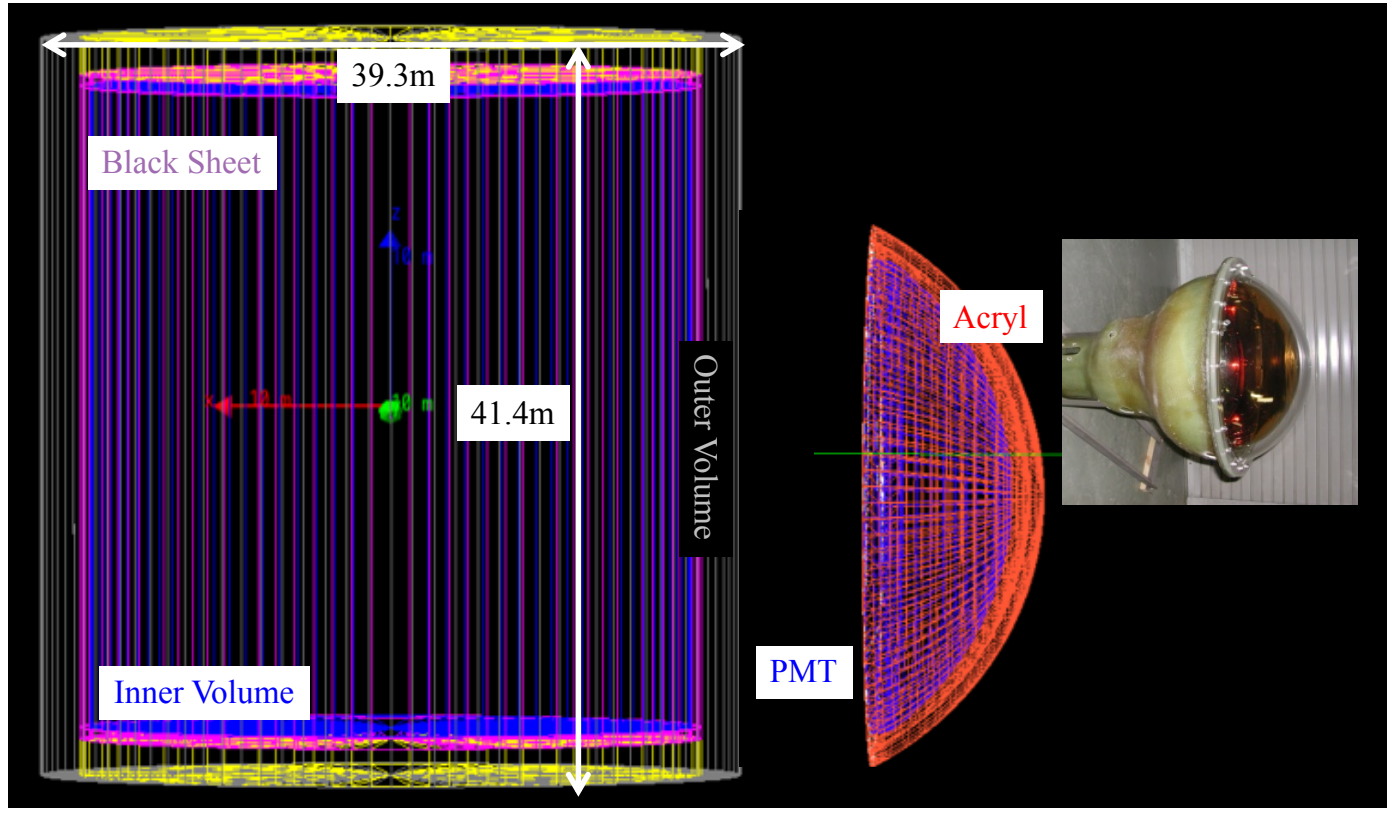

Figure 2: Reproduced Super-K detector geometry. Left figure is overall of detector, right figure is the PMT's.

\subsection{Electromagnetic(EM) physics}

Electromagnetic process is one of the most important for Super-K physics due to that the Cherenkov photon emission which is detected in Super-K is managed in this physics process. SKG4 adopted the Livermore physics list[ $[\mathbb{}]$. The number of emitted Cherenkov photons and hit on PMT was compared with SKDETSIM by generating $10 \mathrm{MeV}$ electron from the center of the tank. Any optical property of photon was not considered in this situation. Table $\square$ shows the result of both simulation. The difference of $2.6 \%$ was seems to be due to the difference in physics model that describe Cherenkov emission.

\begin{tabular}{c|c|c} 
& Number of generated photons & Number of Photons which arrive PMT \\
\hline SKDETSIM & 1948 & 760 \\
SKG4 & 1899 & 740 \\
\hline \hline Ratio & $2.6 \%$ & $2.6 \%$
\end{tabular}

Table 1: Comparison of cherenkov photon emitted from $10 \mathrm{MeV}$ electron between SKG4 and SKDETSIM.

\subsection{Optical photon physics}

Optical photon process, which is including Cherenkov photon is also important. This process manages 3 kinds of optical photon physics, absorption and scattering, behavior in boundary of material. Absorption physics and boundary behavior on the black sheet and PMT were compared. 


\subsubsection{Absorption effect in the water}

Figure 13 shows before and after considering the absorption effect. The number of hits on PMT decreased about $29 \%$ due to the absorption.

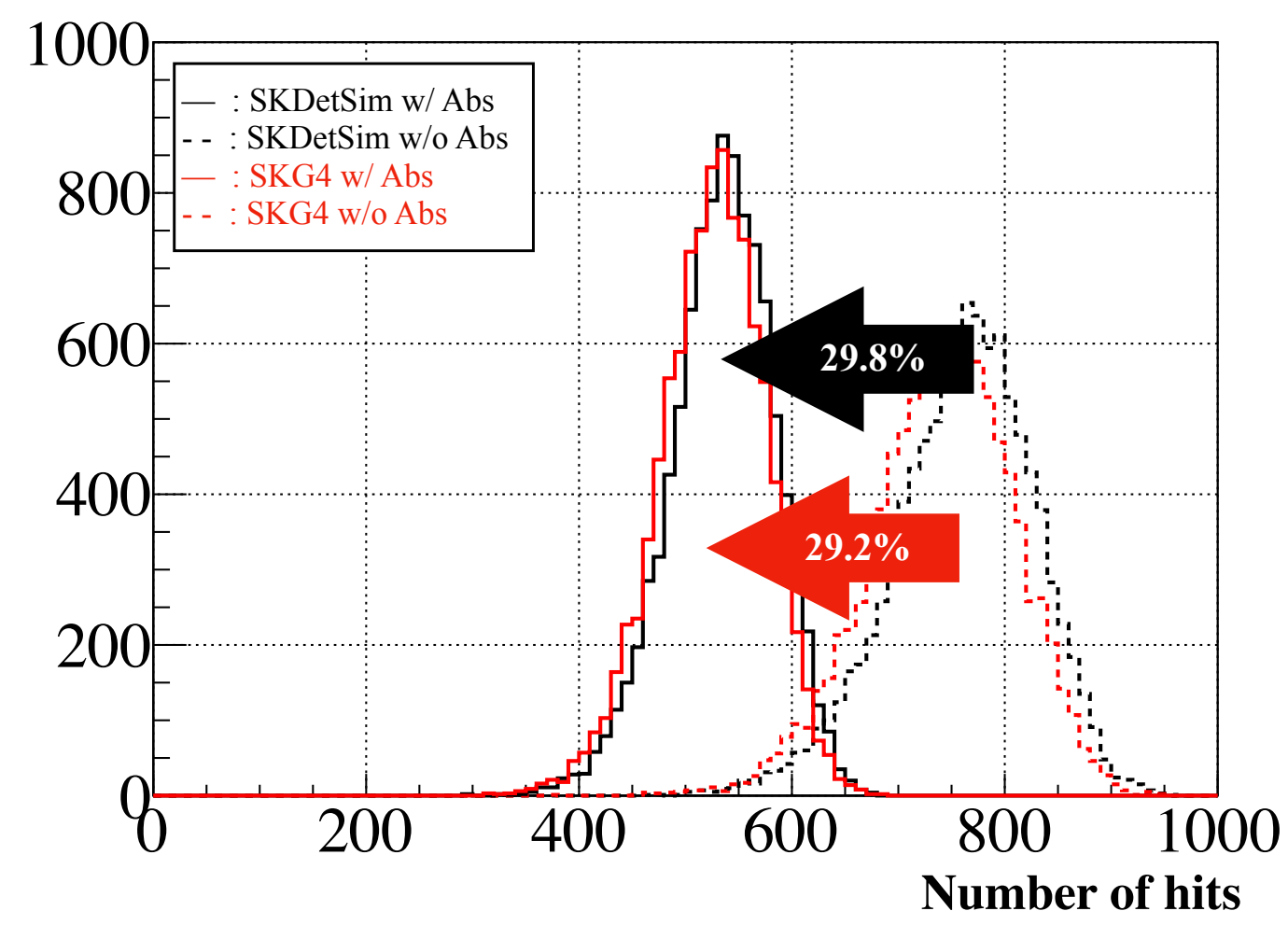

Figure 3: Number of PMT hit with(solid line) or without(dashed line) absorption of water. Red line shows SKG4, black line shows SKDETSIM. The mean value of the distribution is decreased about $29 \%$

\subsubsection{Reflection effect on the black sheet}

The inner wall of tankis covered the black sheet, which has low reflectivity and high absorption probability for optical photon. It should be confirmed that the reflection effect is correctly implemented in SKG4. As method of confirmation, the optical photon with wavelength $350 \mathrm{~nm}$ were generated at center of the tank to random direction, and the number of photon which arrive the PMT surface is counted. The ratio of number of photons arriving at PMT, between the case of with and without black sheet reflection were compared with SKDETSIM. If the black sheet has reflection effect, the number of arriving PMT will be higher than the case of only absorption on the black sheet. Table $\square$ shows the result of 1000000 photons event comparison with SKDETSIM. These values show the number of photon which arrived PMT. As the result, the ratio showed good agreement with SKDETSIM, therefore, it was found that the black sheet reflection method was correctly implemented.

\subsubsection{Reflection and Photoelectric effect on the PMT}

Super-K PMT has Quantum-Efficiency (QE) depended on wavelength. This is up to about 


\begin{tabular}{c|c|c} 
& SKDETSIM & SKG4 \\
\hline Without black sheet reflection & $420500 \pm 649$ & $420447 \pm 648$ \\
With black sheet reflection & $427734 \pm 654$ & $428656 \pm 655$ \\
\hline \hline Ratio of (with/without) black sheet reflection & $101.7 \pm 0.3 \%$ & $101.9 \pm 0.3 \%$
\end{tabular}

Table 2: Comparison of increasing ratio of the number of photons arriving PMT by the reflection effect.

$22 \%$ at wavelength $390 \mathrm{~nm}$. It also has reflection and absorption on the surface. It should be confirmed that the reflection effect is correctly implemented in SKG4. Same method as above was used for confirmation of these effect. The ratio of number of photoelectrons between the case of with and without these were compared with SKDETSIM.

Table [] shows the result of 100000 photons event comparison with SKDETSIM. These value shows the number of photoelectrons. As the result, the ratio shows good agreement with SKDETSIM, therefore, it was found that the PMT response method was correctly implemented.

\begin{tabular}{c|c|c} 
& SKDETSIM & SKG4 \\
\hline Without PMT reflection and QE & $40804 \pm 202.0$ & $41037 \pm 202.5$ \\
With PMT reflection and QE & $7554 \pm 86.9$ & $7579 \pm 87.1$ \\
\hline \hline Ratio of (with/without) PMT reflection and QE & $18.5 \pm 1.6 \%$ & $18.5 \pm 1.6 \%$
\end{tabular}

Table 3: Comparison of ratio of the number of photoelectron by the reflection and QE effect.

\subsection{Result of comparison about EM and optical physics}

To confirm the all effects, behavior of Cherenkov light, absorption, scattering, reflection, and PMT QE, $10 \mathrm{MeV}$ electron was used. The number of photoelectrons were compared between both of simulation. In the above of figure $⿴$ 1 shows the distribution of wavelength of Cherenkov photon emitted from the $10 \mathrm{MeV}$ electron. In this comparison, various wavelength photons can be checked.

In the below figure shows the ratio between both simulation about the wavelength of Cherenkov photon. The difference of the number of Cherenkov photons with SKDETSIM is depends on the wavelength, the difference is up to 5\%. This difference comes from the algorithm of calculation of wavelength and SKG4 is more realistic. Since the behavior of optical photons depends on the wavelength, the difference was appeared in the comparison about Cherenkov photon.

Table $\mathbb{\theta}$ shows the number of generated photons and photoelectrons. The difference of ratio with SKDETSIM between photon and photoelectron can be explained by the difference of distribution about wavelength of Cherenkov photon.

\subsection{1 $10 \mathrm{MeV}$ gamma ray comparison}

$10 \mathrm{MeV}$ gamma ray was compared with SKDETSIM. Figure 1 shows the distribution of the number of photoelectrons. The mean value of SKDETSIM was about $1.6 \%$ higher than the value of SKG4. This is the reasonable value with electron's difference, and this difference can be corrected by gain tune of PMT. 

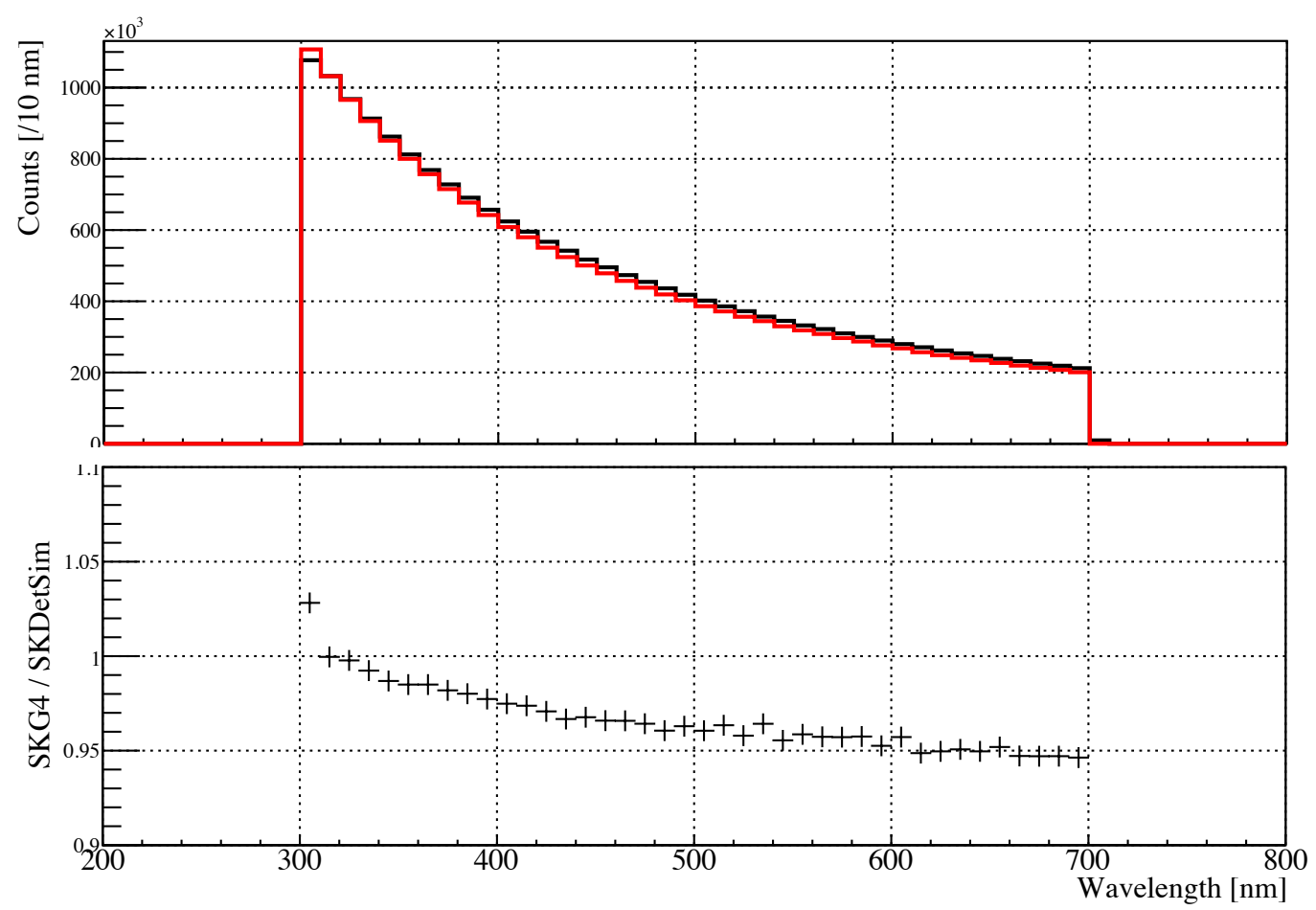

Figure 4: Distribution of the wavelength of Cherenkov photon. Red line shows SKG4, black line shows SKDETSIM.

\begin{tabular}{c|c|c} 
& Number of generated photons & Number of Photons which emit photoelectron \\
\hline SKDETSIM & 1950 & 88.82 \\
SKG4 & 1897 & 87.42 \\
\hline \hline Ratio & $2.7 \%$ & $1.6 \%$
\end{tabular}

Table 4: Comparison of photoelectron between SKG4 and SKDETSIM.

\section{Conclusion and Status}

SK-Gd experiment will start in 2019. Toward SK-Gd, new simulation software named SKG4 was constructed. Geometry of Suepr-K was reproduced with reference to the current simulation, SKDETSIM. In this article, the behavior of the charged particles and optical photons in SKG4 was compared with SKDETSIM. The difference of number of emitted Cherenkov photon by 10 $\mathrm{MeV}$ electron with SKDETSIM was 2.6\%. Difference of reduction ratio by absorption effect from SKDETSIM was $0.6 \%$. The effect of Black sheet, PMT surface got a good agreement with SKDETSIM within $1 \%$. Finally, the check about the number of photoelectron including whole effect got a good agreement within $2.6 \%$. Gamma ray behavior was also evaluated. The difference of photoelectron from the $10 \mathrm{MeV}$ gamma ray is about 1.6\% from SKDETSIM. At present, comparison and tuning by using real Super-K calibration data is in progress. After dissolving Gd in Super-K water, SKG4 will be used various analysis in SK-Gd. 


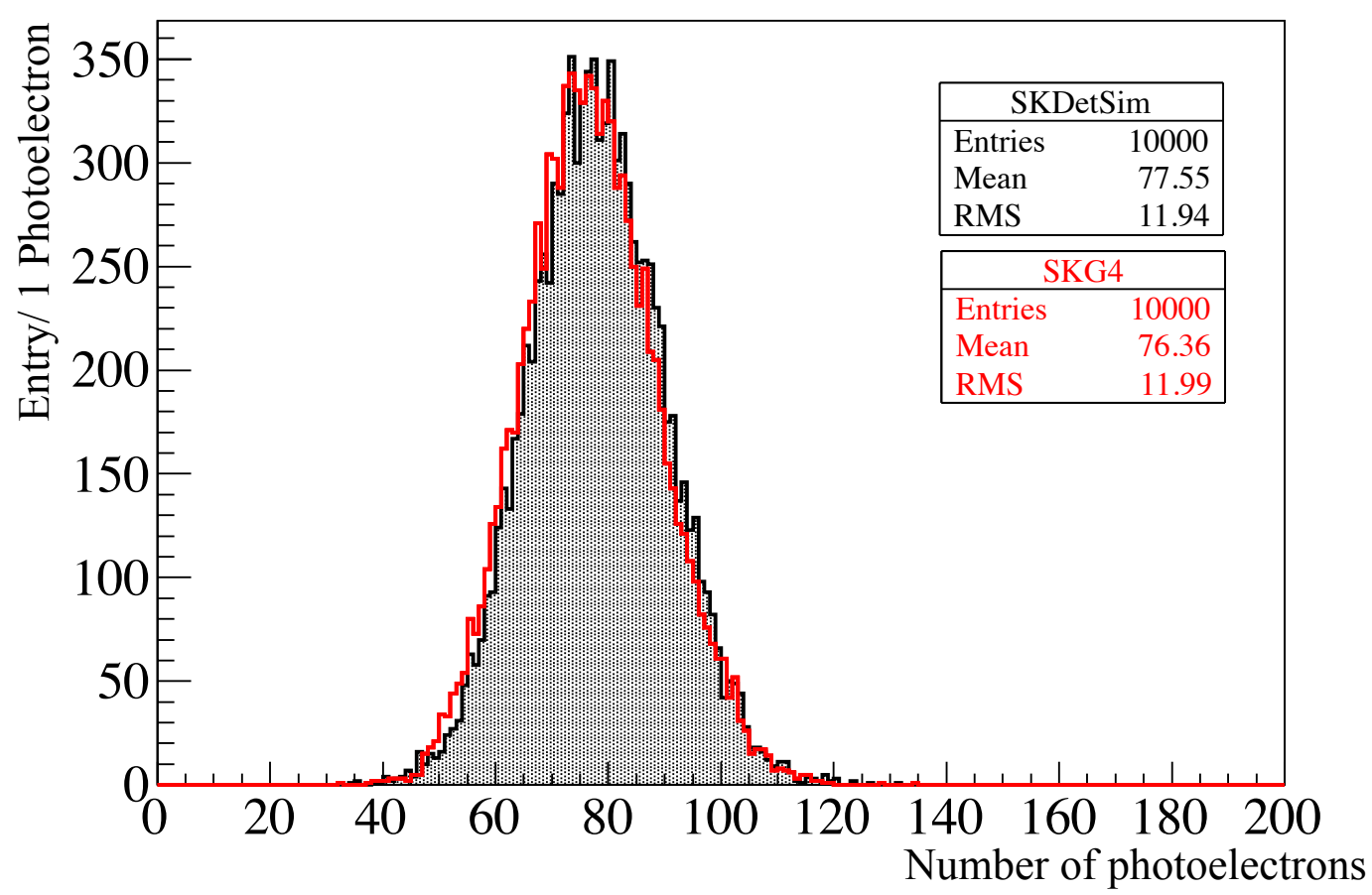

Figure 5: Distribution of the number of photoelectron. Red line shows SKG4, black line shows SKDETSIM.

\section{Acknowledgments}

We gratefully acknowledge cooperation of the Kamioka Mining and Smelting Company. The Super-Kamiokande experiment was built and has been operated with funding from the Japanese Ministry of Education, Science, Sports and Culture, and the U.S. Department of Energy.

\section{References}

[1] K. Bays et al., Phys. Rev. D 85, 052007 (2012).

[2] H. Zhang et al., Astropart. Phys. 60, 41-46 (2015), arXiv:1311.3738.

[3] J. F. Beacom and M. R. Vagins, Phys. Rev. Lett., 93, 171101 (2004).

[4] R. Brun, F. Bruyant, M. Maire, A. C. McPherson and P. Zanarini, CERN-DD-EE-84-1 (1987).

[5] S. Agostinelli et al., Nucl. Instrum, Meth. A506 (2003).

[6] K.Hagiwara et al., Prog. Theor. Exp. Phys. (2018), arXiv:1809.02664v1.

[7] J. Apostolakis et al., Technical Report, CERN/INFN, CERN-OPEN-99-034(1999), INFN/AE-99/18..... 